\title{
Performance Evaluation of Memory-less and Kalman-based Channel Estimation for OFDMA
}

\author{
Daniel Aronsson \\ Dept. of Signals and Systems \\ Uppsala University \\ Email: dar@signal.uu.se
}

\author{
Tommy Svensson \\ Dept. of Communications Systems \\ Chalmers University of Technology \\ Email: tommy.svensson@chalmers.se
}

\author{
Mikael Sternad \\ Dept. of Signals and Systems \\ Uppsala University \\ Email: ms@signal.uu.se
}

\begin{abstract}
The next generation wireless systems based on Orthogonal Frequency Division Multiple Access (OFDMA) need to operate in widely different deployment and usage scenarios. Thus, support for flexible resource allocation is important. In this paper we investigate the performance of different memoryless and memory-based channel estimators for different OFDMA subcarrier allocation schemes and different pilot patterns. We evaluate the performance in various fading environments and for different user terminal velocities. The results show that channel estimation can perform well enough for time-frequency localized resources as small as 22 channel symbols with two pilots in many important scenarios. The results provided can be used to identify appropriate subcarrier allocations for the next generation OFDMA based wireless systems.
\end{abstract}

\section{INTRODUCTION}

Orthogonal Frequency Division Multiple Access (OFDMA) is a promising multiple access technique for future mobile communication systems because of its potential for high spectral efficiency. In a wireless multiuser system, mobile users are subjected to frequency selective and time varying (fading) radio channels. In some situations such as high SINR and modest vehicular speeds, the channel quality can be accurately predicted to enable utilization of the channel frequency selectivity and time-variability for link adaptation and multiuser scheduling gains [1]. In other scenarios such as low SINR, high speeds and multicast transmission, the multi-path fading channel has to be mitigated by diversity-based transmission techniques to obtain robust transmission with low outage probability. Frequency diversity is enhanced by allocating distributed subcarriers for each user, since the fading of these resources then becomes almost uncorrelated. However, under such circumstances a channel estimator cannot take advantage of frequency correlation in the channel estimation procedure. Thus, there is a trade-off for the overall performance of a diversity based OFDMA system between diversity gains and channel estimation performance. The performance of channel estimation depends on the acceptable pilot overhead and deployed channel estimation algorithm, which can be limited by computational complexity.

In the EU FP6 WINNER II project, a Discrete Fourier Transform (DFT) precoded distributed subcarrier scheme has been defined, denoted Block Interleaved Frequency Division Multiple Access (B-IFDMA), for the diversity-based uplink mode and the corresponding scheme for the downlink without
DFT precoding is denoted Block Equidistant Frequency Division Multiple Access (B-EFDMA) [2], [3]. The channel estimation performance for these schemes has been studied within the WINNER II project assuming a frequency multiplexed pilot grid and 2D Wiener filter based channel estimation, [4], [5], [6]. With a dedicated frequency multiplexed pilot grid, the channel estimation problem becomes the same for both schemes.

In this paper we complement the results in [4], [5], [6] by exploring channel estimation performance without memory (Block-Least Squares Estimation, Block-LSE) and channel estimation with memory (Kalman filtering, KF), with and without smoothing, assuming frequency multiplexed pilots. We compare different channel estimators in terms of their ability to correctly recover channel transfer function (CTF) coefficients under different subcarrier allocation schemes and different pilot patterns. The performance is evaluated in various fading environments and for different user terminal velocities. The overall goal of the investigations in this paper is to guide the selection of a proper subcarrier allocation and pilot pattern for diversity-based OFDMA systems in the different evaluated scenarios.

\section{SYSTEM MODEL}

Orthogonal Frequency Division Multiplexing (OFDM) divides the radio channel resource into a time-frequency grid. In OFDMA, different subcarriers and/or OFDM symbols can be allocated to different users. The way resources are assigned to users is here referred to as the block allocation (BA).

Six different BAs proposed by the WINNER II project for diversity-based transmission are studied [3]. All BAs investigated here are variants of B-IFDMA and B-EFDMA, presented in [2], [3]. Five of the six BAs use a 4-subcarriersby-3-OFDM-symbols block as smallest common unit, denoted a basic block. These BAs, which we call B-IFDMA 1x1, BIFDMA 1x2, B-IFDMA 2x1, B-IFDMA 2x2, and LFDMA, assign to each user, at each scheduling slot, multiple 'bins' consisting of one basic block, two basic blocks stacked in frequency, two basic blocks stacked in time, two-by-two basic blocks, and two-by-four basic blocks, respectively. In IFDMA, each bin consists of one single subcarrier with 12 OFDM symbols. 


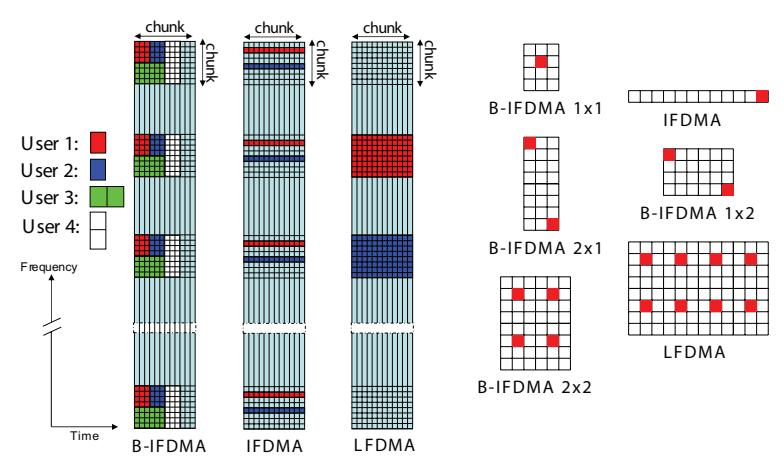

Fig. 1. Illustration of investigated block allocations. The left side shows examples of allocations for multiple users. B-IFDMA 1x1, B-IFDMA 1x2, and B-IFDMA $2 \times 1$ are represented by users 1,3 , and 4 in the far left figure, respectively. (B-IFDMA $2 \times 2$ is not presented in the left figure.) The right side shows optimal or near-optimal pilot patterns as proposed in Section IV.

At each scheduling slot, each user is allocated one or more bins, which are allocated equidistantly in frequency with a spacing considerably larger than the channel coherence bandwidth. See Figure 1. The data rate for each user can thus be adjusted by varying the bin size and the number of bins per scheduling slot. To efficiently support robust transmission of small packets, a small bin size is desirable. As shown in [2, Figure 3], there is a large diversity gain for B-IFDMA and IFDMA compared to LFDMA when perfect channel estimation is assumed.

While small bins scattered over a large portion of the frequency band ensures data integrity, it impedes channel estimation; all frequency taps within a bin are fairly well correlated, but the correlation between frequency taps in different bins is small. Hence we would expect B-IFDMA 1x1 and IFDMA to yield a comparably poor channel estimation performance while LFDMA should give good estimation results.

The bins are also separated in time. We assume half-duplex, so that each scheduling slot consists of 12 OFDM symbols for the uplink, followed by 12 OFDM symbols for the downlink. The scheduling slot period is therefore 24 OFDM symbols which means that there is, e.g., a gap of 18 OFDM symbols between consecutive B-IFDMA 1x1 blocks, but only a gap of 12 OFDM symbols between consecutive LFDMA blocks.

In this paper we assume the use of persistent scheduling in the uplink, which means that the resource allocation for any given user is static and does not change over time. By assuming so, we guarantee that measurements of past bins are always available, so that Kalman-based channel estimators have historic data to operate on ${ }^{1}$. Eight 12 -symbols-blocks are allocated per user at each scheduling slot. This means that eight bins per slot are allocated when B-IFDMA 1x1 and IFDMA are used, and one bin per slot is allocated when LFDMA is used.

\footnotetext{
${ }^{1}$ No such assumption is required for the downlink, since pilots from other users may then be used. Also, the present investigation can be seen as a special case of adaptive scheduling.
}

The system parameter values that we use are those proposed by the WINNER project for urban base coverage ([7]); the FFT bandwidth is $80 \mathrm{MHz}$ divided on 2048 subcarriers, yielding a subcarrier spacing of approximately $39 \mathrm{kHz}$ and an OFDM symbol duration of $25.6 \mu \mathrm{s}(+3.2 \mu$ s for the cyclic prefix $)$. The carrier frequency is set to $3.7 \mathrm{GHz}$.

If the cyclic prefix is at least as long as the channel's delay spread, and if the influences from non-linear system components are negligible, then inter-carrier and inter-symbol interference is avoided. The received signal $\mathbf{y}_{t}$ over a portion of $W$ simultaneous subcarriers can then be modelled by

$$
\mathbf{y}_{t}=\Phi_{t} \mathbf{h}_{t}+\mathbf{v}_{t}
$$

where $W$ is 1,4 , or 8 depending on which BA is used. The $W$-by- $W$ diagonal matrix $\Phi_{t}$ holds the symbols transmitted over the $W$ subcarriers, the $W$-vector $\mathbf{h}_{t}$ is the fading CTF coefficients that we seek to estimate, and the $W$-vector $\mathbf{v}_{t}$ is white additive gaussian noise with variance $N_{0} / 2$ in the real and imaginary parts, respectively. Choosing the number $W$ of parallel subcarriers to take into each measurement is a tradeoff between complexity and performance; measuring many subcarriers at a time allows the channel estimator to take frequency correlation into account which improves the estimate, but it also increases numerical complexity.

The frequency-domain CTF coefficients $\mathbf{h}_{t}$ are modelled on block-diagonal state-space form:

$$
\begin{aligned}
\mathbf{x}_{t+1} & =F \mathbf{x}_{t}+G \mathbf{u}_{t}, \\
\mathbf{h}_{t} & =H \mathbf{x}_{t},
\end{aligned}
$$

where the block-diagonal matrices $F, G$, and $H$ each consist of $W$ blocks. Each such triplet $\left\{F_{w}, G_{w}, H_{w}\right\}$ of blocks (or submatrices) is a model of order $K$ that models one frequencydomain CTF coefficient, which we here call a frequency tap. In this paper we assign a time-invariant model, thus assuming static velocity and Doppler spectrum.

The frequency tap model, the same for all $W$ frequency taps, is set up in such a way that it accommodates a certain Doppler spectrum. We study two different fading statistics. One, which we term rich fading, means to represent a scenario typical for cities, where sideways reflexes are generated by nearby buildings. The other, which we call Jakes-like fading, represents sub-urban conditions with the mobile user surrounded by many equidistant scatterers. It is generally easier to perform channel estimation in the Jakes-like case than in rich fading. The two assumed Doppler spectra are plotted in Fig. 2. The velocity is set to $50 \mathrm{~km} / \mathrm{h}$ in the figure, giving a practical maximum Doppler frequency of just below $200 \mathrm{~Hz}$ although the models will allow some amount of higher frequencies.

The correlation between subcarriers, which is crucial to channel estimation performance, is described by the channel model. The model used here is the WINNER II C2 non-lineof-sight power delay profile (PDP)[8]:

\begin{tabular}{|c|c|}
\hline delay[ns] & \multicolumn{1}{|c|}{ power[dB] } \\
\hline $0,60,75,145,150,155,150$, & $-6.4,-3.4,-2.0,-3.0,-5.2,-7.0,-1.9$, \\
$190,220,225,230,335,370$, & $-3.4,-3.4,-5.6,-7.4,-4.6,-7.8,-7.8$, \\
$430,510,685,725,735,800$, & $-9.3,-12.0,-8.5,-13.2,-11.2,-20.8$, \\
$960,1020,1100,1210,1845$ & $-14.5,-11.7,-17.2,-16.7$ \\
\hline
\end{tabular}




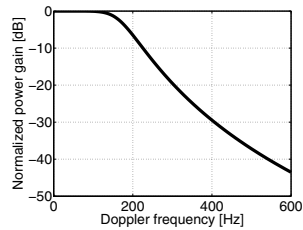

(a) Rich fading

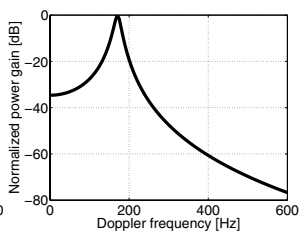

(b) Jakes-like fading
Fig. 2. Doppler spectra for the two fading scenarios.

These correlation properties are built into the channel model by scaling the generally full $W$-by- $W$ covariance matrix for the process noise $\mathbf{u}_{t}$ in the model (2) in such a way that the covariance for the frequency taps $\mathbf{h}_{t}$ is the Fourier transform of the PDP.

\section{Pilot-aided Channel estimation}

Pilot-aided channel estimation (PACE) inserts known symbols, called pilots, into the time-frequency grid. The specification of where each pilot is located is referred to as the pilot pattern. By considering only the pilots in the measurements, disregarding all the payload symbols, the matrix $\Phi_{t}$ in (1) becomes known but time-variant. It is of interest to keep the pilot overhead, i.e. the ratio between number of pilots and the total number of symbols, low. In all the BAs that we study here, the pilot overhead is $1 / 12$.

Non-smoothed KF: The state-space model (2) enables us to directly write down the optimal Kalman observer of the states $\mathbf{x}_{t}$ (see [9]):

$$
\hat{\mathbf{x}}_{t \mid t}=\underbrace{\left(I-K_{f, t} H\right) F}_{A_{t}} \hat{\mathbf{x}}_{t-1 \mid t-1}+K_{f, t} \mathbf{y}_{t} .
$$

The so called filtered state estimate $\hat{\mathbf{x}}_{t \mid t}$ is the optimal least squares estimate given all measurements up to time $t$. We assume in this paper that data has been received for a considerable period of time (persistent scheduling). Enough pilot data has then been received that the channel estimator has settled to a steady state. The Kalman gain $K_{f, t}$, which generally is time-dependent and needs to be updated by a computationally demanding Riccati equation, will then be periodic-constant since the pilot matrix $\Phi_{t}$ is periodic. The periodic series of matrices $\left\{K_{f, t}\right\}$ may therefore be precomputed. For the same reason, the matrix $A_{t}$ is also known. Once the states estimate has been computed, CTF estimates are calculated by

$$
\hat{\mathbf{h}}_{t \mid t}=H \hat{\mathbf{x}}_{t \mid t} .
$$

Smoothed KF: The KF estimate (3) may be improved upon by smoothing the estimates. Given that we are interested in estimating the channel over a block of $W$ subcarriers and $T=t_{2}-t_{1}$ OFDM symbols at any given time, and that we have received all measurements $\mathbf{y}_{t}\left(t_{1}+1<t \leq t_{2}\right)$ over this whole block, we may use data all the way to the end of the block to produce the smoothed estimates $\hat{\mathbf{h}}_{t_{1}+1 \mid t_{2}}, \hat{\mathbf{h}}_{t_{1}+2 \mid t_{2}}, \ldots, \hat{\mathbf{h}}_{t_{2} \mid t_{2}}$ instead of $\hat{\mathbf{h}}_{t_{1}+1 \mid t_{1}+1}, \hat{\mathbf{h}}_{t_{1}+2 \mid t_{1}+2}, \ldots, \hat{\mathbf{h}}_{t_{2} \mid t_{2}}$ as the non-smoothed KF would produce. Smoothed Kalman estimates can be computed from the one-step predictions $\hat{\mathbf{x}}_{t \mid t-1}$, which are computed with the non-smoothed KF:

$$
\hat{\mathbf{x}}_{t \mid t_{2}}=\hat{\mathbf{x}}_{t \mid t-1}+\sum_{j=t}^{t_{2}} B_{j}\left(\mathbf{y}_{j}-H \hat{\mathbf{x}}_{j \mid j-1}\right),
$$

where the matrices $B_{j}$ may be precomputed here (see [9]).

Block-LSE: The last channel estimation algorithm that we examine is the most commonly suggested in the literature. It conducts channel estimation block-wise, basing the estimates only on the measurements taken from the block. Such algorithms go by many names ${ }^{2}$; we shall here refer to it as the block-wise least squares estimate (Block-LSE). It is a common result in linear estimation ([9]); given two gaussian vectors $\mathbf{h}$ and $\mathbf{y}$ with cross-covariance $R_{h y}$ and the latter with covariance $R_{y}$, the optimal least-squares estimate of $\mathbf{h}$ given $\mathbf{y}$ is

$$
\hat{\mathbf{h}}=\mu_{h}+R_{h y} R_{y}^{-1}\left(\mathbf{y}-\mu_{y}\right),
$$

where $\mu_{h}$ and $\mu_{y}$ are the mean values of $\mathbf{h}$ and $\mathbf{y}$ which we here set to all-zeros. The matrices $R_{h y}$ and $R_{y}$ are relatively easily derived from the models (1) and (2) by setting up an extended state-space over all the time steps in the block.

Of the three estimation methods, smoothed Kalman will always have the best performance, since it uses all past measurements. Block-LSE and non-smoothed KF will outperform one another depending on situation; while Block-LSE only uses local data and keeps no record of measurements of previous bins, non-smoothed KF learns from history. However, BlockLSE will use all data that it has available even for estimating the frequency taps located early in the bin. As a contrast, non-smoothed KF will generally give poor performance for early frequency taps, since it at that point has only historic measurements to base its decision on.

\section{Simulation Results}

The BAs are tested for two different fading statistics (see Sec. II) and three different velocities: $5 \mathrm{~km} / \mathrm{h}, 50 \mathrm{~km} / \mathrm{h}$, and $250 \mathrm{~km} / \mathrm{h}$. Perfect model match is assumed, so that the model (2) correctly represents the real channel. We also assume persistent scheduling with stationary conditions (that is, a static model) in which transients from earlier conditions have faded.

The algorithms are tested over a range of symbol-energy-tonoise-spectral-density ratios $\left(E_{s} / N_{0}\right)$, and measured in terms of the resulting signal-to-error ratios (SER). The total error contributed by the $E_{s} / N_{0}$ and the SER ultimately leads to a certain bit error rate (BER) or a certain frame error rate (FER). The ideal BER/FER curve is that produced when $\mathrm{SER}=\infty$. When $\mathrm{SER}=E_{s} / N_{0}$, the estimation error 'power' equals the noise power, so that the total SER+SNR is $3 \mathrm{~dB}$ below SNR. The BER/FER value is then displaced $3 \mathrm{~dB}$ from the ideal curve. However, results in [6] and [5] suggest that this displacement can be improved down to a mere 1 $\mathrm{dB}$ displacement when iterative channel estimation (ICE) is

\footnotetext{
${ }^{2}$ The name Wiener filters is common in the literature. That notion however has ambiguous meaning so we refrain from using it here.
} 


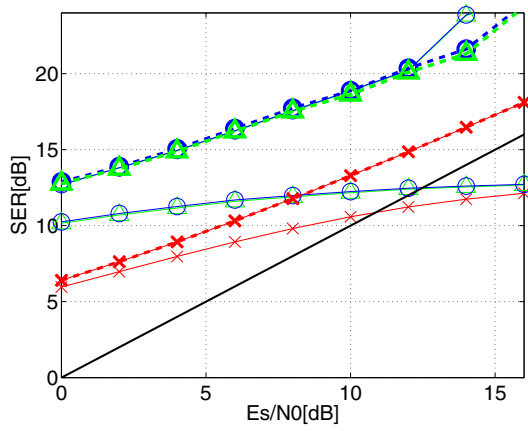

(a) $5 \mathrm{~km} / \mathrm{h}$, Jakes-like fading

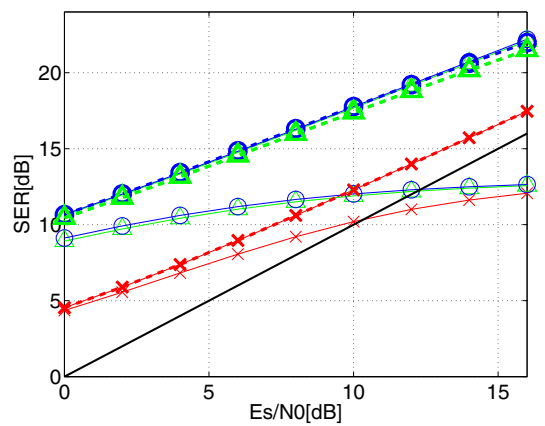

(d) $5 \mathrm{~km} / \mathrm{h}$, rich fading

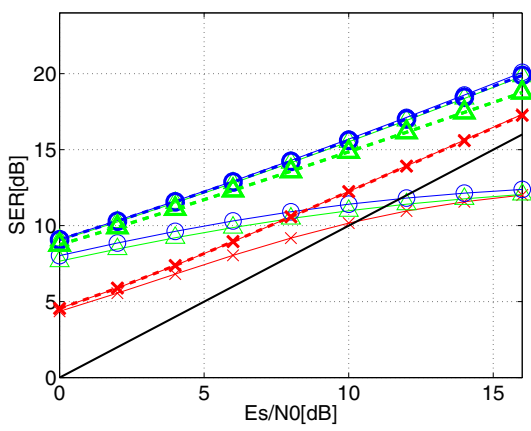

(b) $50 \mathrm{~km} / \mathrm{h}$, Jakes-like fading

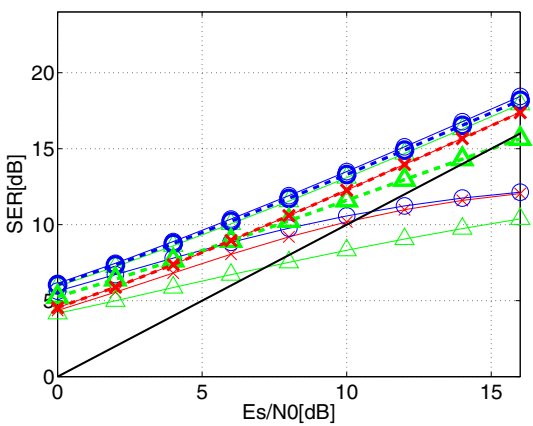

(e) $50 \mathrm{~km} / \mathrm{h}$, rich fading

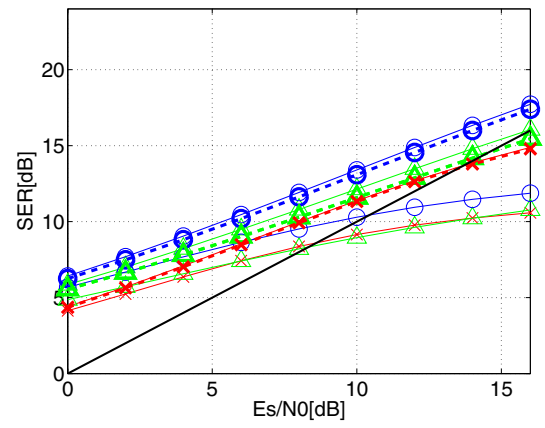

(c) $250 \mathrm{~km} / \mathrm{h}$, Jakes-like fading

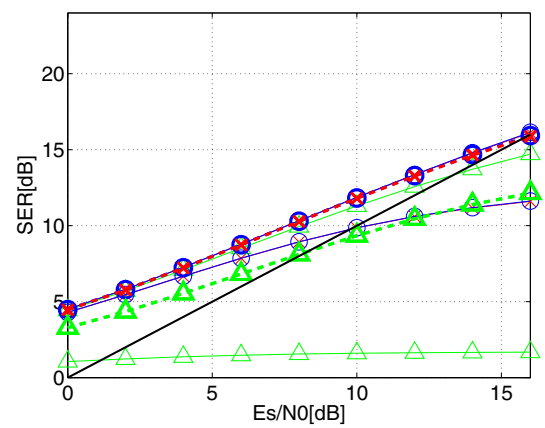

(f) $250 \mathrm{~km} / \mathrm{h}$, rich fading

Fig. 3. Estimation performance for B-IFDMA 2x1-mode at different velocities and with different fading statistics. Simulations were carried out over every possible two-pilots pattern. Worst and best performance curves are indicated by thin solid lines for Kalman filtering (KF) with smoothing (blue, circles), KF without smoothing (green, triangles), and block-LSE (red, crosses). Thick dashed lines indicate performance for the 'opposite corners' pattern. The solid line without markers is the acceptance boundary $\mathrm{SER}=E_{s} / N_{0}$.

used. Motivated by this, we use $\mathrm{SER}=E_{s} / N_{0}$ as criterion for acceptable channel estimation performance.

The 36 scenarios that we have investigated are schematically illustrated in Figure 5. Unfortunately, space limitations pre-

\begin{tabular}{|c|c|c|c|c|c|c|}
\hline & & & & $M_{A_{2}}$ & \multicolumn{2}{|c|}{${ }_{A} \delta_{M_{A}}$} \\
\hline $5 \mathrm{~km} / \mathrm{h}$, jakes-like fading & & & & $3 a$ & & \\
\hline $5 \mathrm{~km} / \mathrm{h}$, rich fading & & & & $3 d$ & & \\
\hline $50 \mathrm{~km} / \mathrm{h}$, jakes-like fading & & & & $3 b$ & & \\
\hline $50 \mathrm{~km} / \mathrm{h}$, rich fading & $4 a$ & $4 \mathrm{~b}$ & $4 \mathrm{c}$ & $3 e, 4 d$ & $4 e$ & 4 \\
\hline $250 \mathrm{~km} / \mathrm{h}$, jakes-like fading & & & & $3 c$ & & \\
\hline $250 \mathrm{~km} / \mathrm{h}$, rich fading & & & & $3 f$ & & \\
\hline
\end{tabular}

Fig. 5. Summary of 36 combinations and block allocations, velocities, and fading statistics. Dark grey indicates that none of the three channel estimators suggested here meet the performance criterion. Light grey indicates that Block-LSE does not meet the criterion. Detailed results for twelve combinations are presented in other figures, as indicated by the numbers and letters.

vents us from presenting all these results. Instead we present results for both fading statistics and all velocities for just one block allocation (B-IFDMA 2x1), see Figure 3. We also present results for all the six BAs, but only for $50 \mathrm{~km} / \mathrm{h}$ in rich fading, see Figure 4. These choices are indicated in Figure 5. To give an overview of all the results, we indicate in Figure 5 the scenarios that do not meet the performance limit for any of the estimation methods.

\section{A. Impact of pilot pattern}

Although the assumed pilot overhead is fixed to $1 / 12$, it is not evident were exactly the pilots should be placed in the time-frequency grid. We therefore conduct exhaustive searches over all possible patterns in order to find the optimal pattern for each BA. We have also hand-picked one pattern for each BA that performs well in all scenarios. These patterns are illustrated i Figure 1. Exhaustive searches could not be performed for the last two BAs. Instead, 100 random patterns were set to compete.

The channel estimation results are presented in Figures 3 and 4 . The performance for the best and worst pilot patterns for the respective estimation methods are plotted, along with the hand-picked pattern. The main conclusion here is that the choice of pilot pattern has a large impact on filtering performance. The patterns presented in Figure 1 have close to optimal performance in all situations.

\section{B. Impact of terminal velocity and fading statistics}

We examine how terminal velocity and fading statistics affect channel estimation performance. B-IFDMA $2 \times 1$ is chosen as a representative BA. See Figure 3. We find that KF estimation is generally superior to Block-LSE, except for high velocities and rich fading, where smoothed KF and BlockLSE have the same performance. It is also important to use smoothing when using KF for the 'harsher' scenarios $(50 \mathrm{~km} / \mathrm{h}$ with rich fading, and $250 \mathrm{~km} / \mathrm{h}$ ). 


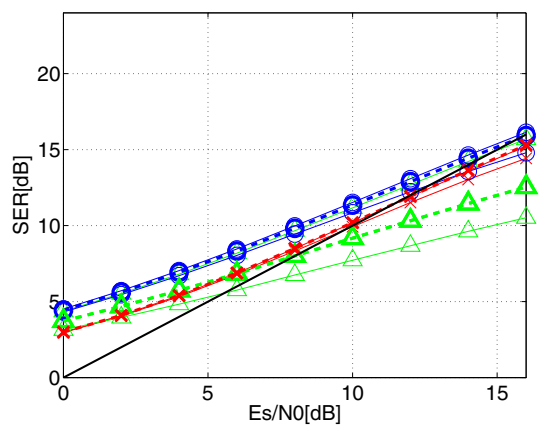

(a) B-IFDMA $1 \times 1$

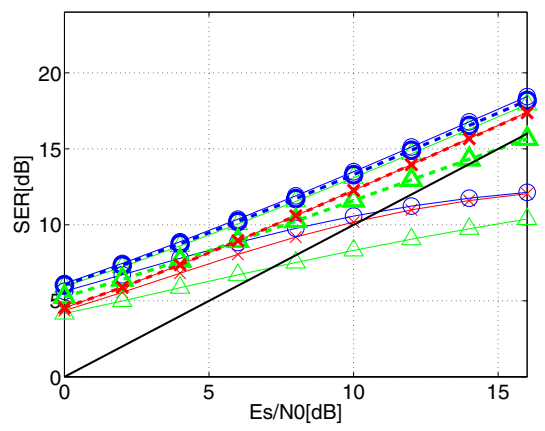

(d) B-IFDMA $2 \times 1$

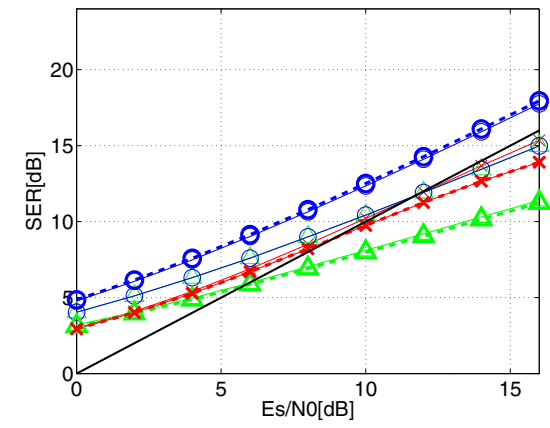

(b) IFDMA

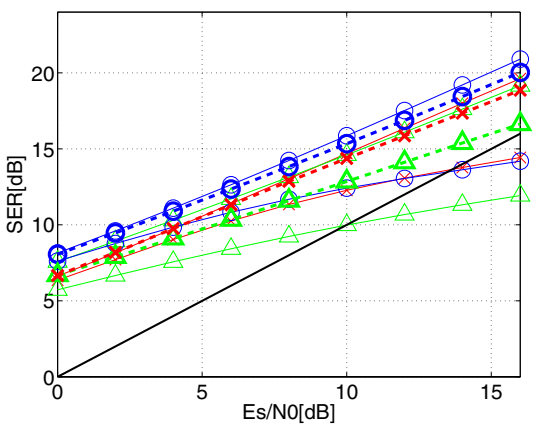

(e) B-IFDMA 2x2

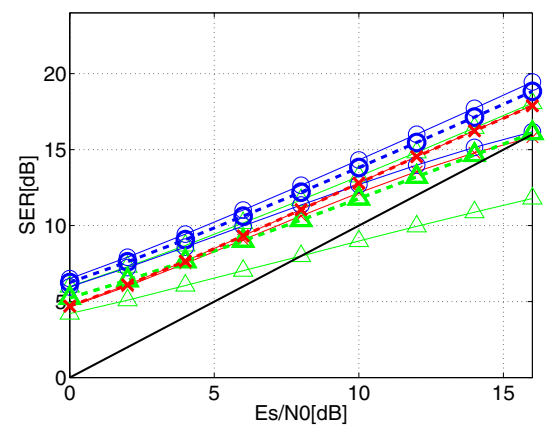

(c) B-IFDMA $1 \times 2$

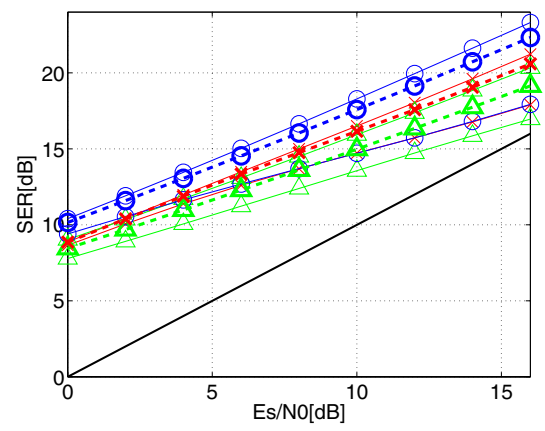

(f) LFDMA

Fig. 4. Estimation performance for the six different block allocations at $50 \mathrm{~km} / \mathrm{h}$ and rich fading. Simulations were carried out over every possible pilot pattern. Worst and best performance curves were picked out and are indicated by thin solid lines for Kalman filtering (KF) with smoothing (blue, circles), KF without smoothing (green, triangles), and block-LSE (red, crosses). The thick dashed lines indicate special pilot patterns that are expected to yield good estimation performance (see Figure 1). The solid line without markers is the acceptance boundary $\mathrm{SER}=E_{s} / N_{0}$.

\section{Impact of block allocation choice}

The choice of block allocation greatly impacts estimation performance. For low velocities, all six BAs considered here meet the performance criterion over the whole SNR range, although KF estimation needs to be used for B-IFDMA $1 \mathrm{x} 1$. However at higher velocities, the performance for BAs using small bins quickly deteriorates, especially in rich fading scenarios. We study the specific case $50 \mathrm{~km} / \mathrm{h}$ and rich fading in Figure 4. Evidently, the use of B-IFDMA 1x1 could be questioned in the studied scenario, and IFDMA may need smoothed KF estimation. Small bins may however be attractive when transmitting small packets in situations when a low outage probability is more important than the power efficiency of the transmission, or in the downlink when pilots from adjacent users can be used. We also note that KF with smoothing must be used for the 24-symbols-per-bin schedules at high velocities in order to meet the performance boundary.

\section{CONClusion}

In this paper we have investigated channel estimation for OFDMA based resource allocation schemes. Within the framework of Block Interleaved Frequency Division Multiple Access (B-IFDMA) we showed that channel estimation can perform well for small time-frequency localized resources, bins. Small bins are useful for robust frequency-diversity based transmission of small packets. Combined with persistent scheduling, we find that smoothed Kalman filtering (KF) estimation is generally superior to Block-LSE, except for high velocities and rich fading, where smoothed $\mathrm{KF}$ and BlockLSE have the same performance. With KF, bins as small as 22 channel symbols and two pilots can be used even at high velocities.

\section{REFERENCES}

[1] M. Sternad, T. Svensson, T. Ottosson, A. Ahlén, A. Svensson, and A. Brunstrom, "Towards systems beyond $3 \mathrm{G}$ based on adaptive OFDMA transmission," Proceding of the IEEE, Special Issue on Adaptive Transmission, vol. 95, no. 12, pp. 2432-2455, Dec 2007.

[2] T. Svensson et al, "B-IFDMA - A Power Efficient Multiple Access Scheme for Non-frequency-adaptive Transmission", IST Mobile and Vehicular Summit, Budapest, July 2007.

[3] IST-4-027756 WINNER II, "D6.13.14 v1.1: WINNER II System Concept Description", Jan. 2008. Available on http://www.signal.uu.se/Publications/wip.html.

[4] IST-4-027756 WINNER II, "D2.3.3: Link level procedures for the WINNER System", Nov. 2007. Available on http://www.signal.uu.se/Publications/wip.html.

[5] C. Lam, D. Falconer, and F. Danilo-Lemoine, "Channel Estimation for Sub-Chunk-based DFT-precoded OFDM Systems", Wireless World Research Forum, Helsinki, June 2007

[6] J. Bonnet and G. Auer, "Chunk-based Channel Estimation for Uplink OFDM", IEEE Vehicular Technology Conference, Melbourne, May 2006

[7] IST-4-027756 WINNER II, "D6.13.7: Test Scenarios and Calibration Cases Issue 2", Dec. 2006. Available on http://www.signal.uu.se/Publications/wip.html.

[8] IST-4-027756 WINNER II, "D1.1.2 v1.2 Part 1: Channel Models", Sep. 2007. Available on http://www.signal.uu.se/Publications/wip.html.

[9] T. Kailath, A.H. Sayed, and B. Hassibi, "Linear Estimation" PrenticeHall, Upper Saddle River, NJ, 2000. 